\title{
Dispersion relation for hadronic light-by-light scattering
}

\author{
Massimiliano Procura ${ }^{1, a}$, Gilberto Colangelo ${ }^{2, b}$, Martin Hoferichter ${ }^{3, c}$, and Peter Stoffer ${ }^{4, d}$ \\ ${ }^{1}$ CERN Theory Division, 1211 Geneva 23, Switzerland \\ ${ }^{2}$ Albert Einstein Center for Fundamental Physics, Institute for Theoretical Physics, University of Bern, Sidlerstrasse 5, 3012 Bern, \\ Switzerland \\ ${ }^{3}$ Institute for Nuclear Theory, University of Washington, Seattle, WA 98195-1550, USA \\ ${ }^{4}$ Helmholtz-Institut für Strahlen- und Kernphysik (Theory) and Bethe Center for Theoretical Physics, University of Bonn, 53115 \\ Bonn, Germany
}

\begin{abstract}
The largest uncertainties in the Standard Model calculation of the anomalous magnetic moment of the muon $(g-2)_{\mu}$ come from hadronic contributions. In particular, in a few years the subleading hadronic light-by-light (HLbL) contribution might dominate the theory uncertainty. We present a dispersive description of the HLbL tensor, which is based on unitarity, analyticity, crossing symmetry, and gauge invariance. This opens up the possibility of a data-driven determination of the HLbL contribution to $(g-2)_{\mu}$ with the aim of reducing model dependence and achieving a reliable error estimate.

Our dispersive approach defines unambiguously the pion-pole and the pion-box contribution to the HLbL tensor. Using Mandelstam's double-spectral representation, we have proven that the pion-box contribution coincides exactly with the one-loop scalar QED amplitude, multiplied by the appropriate pion vector form factors.
\end{abstract}

\section{Introduction}

The anomalous magnetic moment of the muon $(g-2)_{\mu}$ has been measured [1] and computed to very high precision of about $0.5 \mathrm{ppm}$ (see e.g. [2]). For more than a decade, a discrepancy has persisted between the experiment and the Standard Model prediction, now of about $3 \sigma$. Forthcoming measurements at FNAL [3] and J-PARC [4] will update the experimental value. The aim is to increase the precision by a factor of 4 and check for systematic errors.

The main uncertainty of the theory prediction is due to strong interaction effects. At present, the largest error arises from hadronic vacuum polarisation, which, however, forthcoming data from $e^{+} e^{-}$experiments [2] may help reduce. Thus in a few years, the subleading ${ }^{1}$ hadronic light-by-light contribution might dominate the theory error. In present calculations of the HLbL contribution, systematic errors are difficult, if not impossible, to quantify, due to model dependence. A new strategy is required to provide a solid estimate of the theory uncertainties and reduce them. In the recent past, lattice QCD has made remarkable progress in this direction, and may play a leading role in this field in the near future [8-12]. In [13, 14], we have presented the first dispersive description of the

\footnotetext{
a e-mail: massimiliano.procura@cern.ch

be-mail: gilberto@itp.unibe.ch

ce-mail: mhofer@uw.edu

de-mail: stoffer@hiskp.uni-bonn.de

${ }^{1}$ Even higher-order hadronic contributions have been considered in $[5,6]$.
}

HLbL tensor. ${ }^{2}$ By making use of the fundamental principles of unitarity, analyticity, crossing symmetry, and gauge invariance, we provide an approach that reduces model dependence and allows for a more data-driven determination of the HLbL contribution to $(g-2)_{\mu}$.

Here, we report on an improvement of our dispersive framework $[16,17]$. We have constructed a generating set of Lorentz structures for the HLbL tensor that is free of kinematic singularities and zeros. This simplifies significantly the calculation of the HLbL contribution to $(g-2)_{\mu}$. Within our dispersive formalism, the definitions of both the pion-pole and pion-box topologies are unambiguous. By constructing a Mandelstam representation for the scalar functions, we prove that the box topologies are equal to the scalar QED (SQED) contribution multiplied by pion vector form factors. First numerical results for the pion-box topologies are shown and future steps are discussed.

\section{Lorentz structure of the HLbL tensor}

In order to study the HLbL contribution to $(g-2)_{\mu}$, we need a description of the HLbL tensor, namely the hadronic Green's function of four quark electromagnetic currents,

\footnotetext{
${ }^{2} \mathrm{~A}$ different approach, which aims at a dispersive description of the muon vertex function instead of the HLbL tensor, has been presented in [15].
}

(C) The Authors, published by EDP Sciences. This is an open access article distributed under the terms of the Creative Commons Attribution License 4.0 (http://creativecommons.org/licenses/by/4.0/). 
evaluated in pure QCD:

$$
\begin{aligned}
\Pi^{\mu \nu \lambda \sigma}\left(q_{1}, q_{2}, q_{3}\right)= & -i \int d^{4} x d^{4} y d^{4} z e^{-i\left(q_{1} \cdot x+q_{2} \cdot y+q_{3} \cdot z\right)} \\
& \times\left\langle 0\left|T\left\{j_{\mathrm{em}}^{\mu}(x) j_{\mathrm{em}}^{\nu}(y) j_{\mathrm{em}}^{\lambda}(z) j_{\mathrm{em}}^{\sigma}(0)\right\}\right| 0\right\rangle .
\end{aligned}
$$

Gauge invariance requires the HLbL tensor to satisfy the Ward-Takahashi (WT) identities

$$
\left\{q_{1}^{\mu}, q_{2}^{v}, q_{3}^{\lambda}, q_{4}^{\sigma}\right\} \Pi_{\mu \nu \lambda \sigma}\left(q_{1}, q_{2}, q_{3}\right)=0,
$$

where $q_{4}=q_{1}+q_{2}+q_{3}$. The HLbL tensor can be written a priori in terms of 138 basic Lorentz structures built out of the metric tensor and the four-momenta [18]. Our first task is to write the HLbL tensor in terms of Lorentz structures that satisfy the WT identities, while at the same time the scalar functions that multiply these structures must be free of kinematic singularities and zeros. A recipe for the construction of these structures has been given by Bardeen, Tung [19], and Tarrach [20] for generic photon amplitudes. Gauge invariance imposes 95 linear relations between the 138 initial scalar functions. A generating $\operatorname{set}^{3}$ consisting of 43 elements can be constructed following Bardeen and Tung [19]. However, as it was shown by Tarrach [20], such a set is not free of kinematic singularities and has to be supplemented by additional structures. We find a redundant generating set of dimension 54:

$$
\Pi^{\mu \nu \lambda \sigma}\left(q_{1}, q_{2}, q_{3}\right)=\sum_{i=1}^{54} T_{i}^{\mu \nu \lambda \sigma} \Pi_{i}(s, t, u),
$$

where the scalar functions $\Pi_{i}$ are free of kinematic singularities and zeros, and therefore suited for a dispersive description. The Mandelstam variables are defined by $s=\left(q_{1}+q_{2}\right)^{2}, t=\left(q_{1}+q_{3}\right)^{2}, u=\left(q_{2}+q_{3}\right)^{2}$. There

\footnotetext{
${ }^{3}$ In 4 space-time dimensions, there are two additional linear relations, hence a basis consists of 41 elements [21, 22].
}

are only seven distinct Lorentz structures:

$$
\begin{aligned}
& T_{1}^{\mu \nu \lambda \sigma}=\epsilon^{\mu v \alpha \beta} \epsilon^{\lambda \sigma \gamma \delta} q_{1_{\alpha}} q_{2 \beta} q_{3 \gamma} q_{4_{\delta}}, \\
& T_{4}^{\mu \nu \lambda \sigma}=\left(q_{2}^{\mu} q_{1}^{\nu}-q_{1} \cdot q_{2} g^{\mu \nu}\right)\left(q_{4}^{\lambda} q_{3}^{\sigma}-q_{3} \cdot q_{4} g^{\lambda \sigma}\right), \\
& T_{7}^{\mu \nu \lambda \sigma}=\left(q_{2}^{\mu} q_{1}^{\nu}-q_{1} \cdot q_{2} g^{\mu \nu}\right)\left(q_{1} \cdot q_{4}\left(q_{1}^{\lambda} q_{3}^{\sigma}-q_{1} \cdot q_{3} g^{\lambda \sigma}\right)\right. \\
& \left.+q_{4}^{\lambda} q_{1}^{\sigma} q_{1} \cdot q_{3}-q_{1}^{\lambda} q_{1}^{\sigma} q_{3} \cdot q_{4}\right) \text {, } \\
& T_{19}^{\mu \nu \lambda \sigma}=\left(q_{2}^{\mu} q_{1}^{\nu}-q_{1} \cdot q_{2} g^{\mu \nu}\right)\left(q_{2} \cdot q_{4}\left(q_{1}^{\lambda} q_{3}^{\sigma}-q_{1} \cdot q_{3} g^{\lambda \sigma}\right)\right. \\
& \left.+q_{4}^{\lambda} q_{2}^{\sigma} q_{1} \cdot q_{3}-q_{1}^{\lambda} q_{2}^{\sigma} q_{3} \cdot q_{4}\right), \\
& T_{31}^{\mu \nu \lambda \sigma}=\left(q_{2}^{\mu} q_{1}^{\nu}-q_{1} \cdot q_{2} g^{\mu \nu}\right)\left(q_{2}^{\lambda} q_{1} \cdot q_{3}-q_{1}^{\lambda} q_{2} \cdot q_{3}\right) \\
& \times\left(q_{2}^{\sigma} q_{1} \cdot q_{4}-q_{1}^{\sigma} q_{2} \cdot q_{4}\right), \\
& T_{37}^{\mu \nu \lambda \sigma}=\left(q_{3}^{\mu} q_{1} \cdot q_{4}-q_{4}^{\mu} q_{1} \cdot q_{3}\right) \\
& \times\left(q_{3}^{v} q_{4}^{\lambda} q_{2}^{\sigma}-q_{4}^{v} q_{2}^{\lambda} q_{3}^{\sigma}+g^{\lambda \sigma}\left(q_{4}^{v} q_{2} \cdot q_{3}-q_{3}^{v} q_{2} \cdot q_{4}\right)\right. \\
& +g^{\nu \sigma}\left(q_{2}^{\lambda} q_{3} \cdot q_{4}-q_{4}^{\lambda} q_{2} \cdot q_{3}\right) \\
& \left.+g^{\lambda v}\left(q_{3}^{\sigma} q_{2} \cdot q_{4}-q_{2}^{\sigma} q_{3} \cdot q_{4}\right)\right), \\
& T_{49}^{\mu \nu \lambda \sigma}=q_{3}^{\sigma}\left(q_{1} \cdot q_{3} q_{2} \cdot q_{4} q_{4}^{\mu} g^{\lambda \nu}-q_{2} \cdot q_{3} q_{1} \cdot q_{4} q_{4}^{\nu} g^{\lambda \mu}\right. \\
& +q_{4}^{\mu} q_{4}^{\gamma}\left(q_{1}^{\lambda} q_{2} \cdot q_{3}-q_{2}^{\lambda} q_{1} \cdot q_{3}\right) \\
& +q_{1} \cdot q_{4} q_{3}^{\mu} q_{4}^{v} q_{2}^{\lambda}-q_{2} \cdot q_{4} q_{4}^{\mu} q_{3}^{v} q_{1}^{\lambda} \\
& \left.+q_{1} \cdot q_{4} q_{2} \cdot q_{4}\left(q_{3}^{\nu} g^{\lambda \mu}-q_{3}^{\mu} g^{\lambda \nu}\right)\right) \\
& -q_{4}^{\lambda}\left(q_{1} \cdot q_{4} q_{2} \cdot q_{3} q_{3}^{\mu} g^{v \sigma}-q_{2} \cdot q_{4} q_{1} \cdot q_{3} q_{3}^{\nu} g^{\mu \sigma}\right. \\
& +q_{3}^{u} q_{3}^{v}\left(q_{1}^{\sigma} q_{2} \cdot q_{4}-q_{2}^{\sigma} q_{1} \cdot q_{4}\right) \\
& +q_{1} \cdot q_{3} q_{4}^{\mu} q_{3}^{v} q_{2}^{\sigma}-q_{2} \cdot q_{3} q_{3}^{\mu} q_{4}^{v} q_{1}^{\sigma} \\
& \left.+q_{1} \cdot q_{3} q_{2} \cdot q_{3}\left(q_{4}^{\nu} g^{\mu \sigma}-q_{4}^{\mu} g^{v \sigma}\right)\right) \\
& +q_{3} \cdot q_{4}\left(\left(q_{1}^{\lambda} q_{4}^{\mu}-q_{1} \cdot q_{4} g^{\lambda \mu}\right)\left(q_{3}^{\nu} q_{2}^{\sigma}-q_{2} \cdot q_{3} g^{\nu \sigma}\right)\right. \\
& \left.-\left(q_{2}^{\lambda} q_{4}^{v}-q_{2} \cdot q_{4} g^{\lambda \nu}\right)\left(q_{3}^{\mu} q_{1}^{\sigma}-q_{1} \cdot q_{3} g^{\mu \sigma}\right)\right) .
\end{aligned}
$$

All the remaining ones are just crossed versions of the seven structures above. As each structure fullfils the WT identities, both crossing symmetry and gauge invariance are implemented in a manifest way in the set $\left\{T_{i}^{\mu \nu \lambda \sigma}\right\}$.

\section{HLbL contribution to $(g-2)_{\mu}$}

The HLbL contribution to $a_{\mu}=(g-2)_{\mu} / 2$ can be extracted with the help of well-known Dirac projector techniques [23]. With our decomposition of the HLbL tensor in 54 structures, this amounts to the calculation of the following two-loop integral:

$$
\begin{aligned}
a_{\mu}^{\mathrm{HLbL}}= & -\frac{e^{6}}{48 m_{\mu}} \int \frac{d^{4} q_{1}}{(2 \pi)^{4}} \frac{d^{4} q_{2}}{(2 \pi)^{4}} \frac{1}{q_{1}^{2} q_{2}^{2}\left(q_{1}+q_{2}\right)^{2}} \\
& \times \frac{1}{\left(p+q_{1}\right)^{2}-m_{\mu}^{2}} \frac{1}{\left(p-q_{2}\right)^{2}-m_{\mu}^{2}} \\
& \times \operatorname{Tr}\left(\left(\not p+m_{\mu}\right)\left[\gamma_{\rho}, \gamma_{\sigma}\right]\left(\not p+m_{\mu}\right) \gamma_{\mu}\right. \\
& \left.\left(\not p+\not q_{1}+m_{\mu}\right) \gamma_{\lambda}\left(\not p-q_{2}+m_{\mu}\right) \gamma_{\nu}\right) \\
& \times\left.\sum_{i=1}^{54}\left(\frac{\partial}{\partial q_{4 \rho}} T_{i}^{\mu \nu \lambda \sigma}\left(q_{1}, q_{2}, q_{4}-q_{1}-q_{2}\right)\right)\right|_{q_{4}=0} \\
& \times \prod_{i}\left(q_{1}, q_{2},-q_{1}-q_{2}\right) .
\end{aligned}
$$

After a Wick rotation of the momenta, five of the eight loop integrals can be carried out with the technique of 


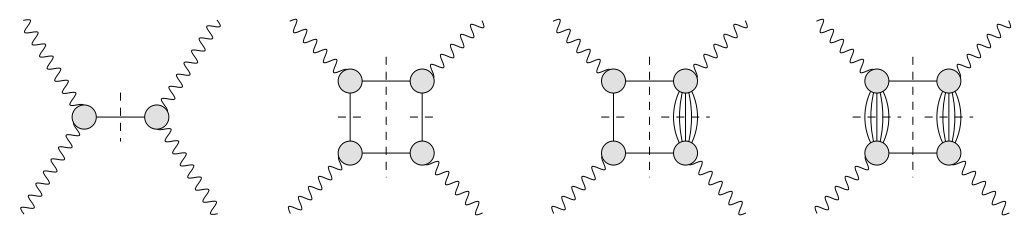

Figure 1. Unitarity diagrams according to the Mandelstam representation. Crossed diagrams are omitted.

Gegenbauer polynomials [24]. We have checked that this Wick rotation is justified even in the presence of anomalous thresholds in the scalar functions $\Pi_{i}$. In analogy to the pion-pole contribution [25], a master formula for the full HLbL contribution to $(g-2)_{\mu}$ can be worked out:

$$
\begin{aligned}
a_{\mu}^{\mathrm{HLbL}}= & \frac{2 \alpha^{3}}{3 \pi^{2}} \int_{0}^{\infty} d Q_{1} \int_{0}^{\infty} d Q_{2} \int_{-1}^{1} d \tau \sqrt{1-\tau^{2}} Q_{1}^{3} Q_{2}^{3} \\
& \times \sum_{i=1}^{12} T_{i}\left(Q_{1}, Q_{2}, \tau\right) \bar{\Pi}_{i}\left(Q_{1}, Q_{2}, \tau\right),
\end{aligned}
$$

where $\alpha=e^{2} /(4 \pi)$ and the $T_{i}$ are integration kernels. With $Q_{i}$ we denote Euclidean momenta. Only twelve independent linear combinations of the hadronic scalar functions $\Pi_{i}$ contribute, denoted by $\bar{\Pi}_{i}[17]$. They have to be evaluated for the reduced kinematics

$$
\begin{aligned}
s & =-Q_{3}^{2}, \quad t=-Q_{2}^{2}, \quad u=-Q_{1}^{2}, \\
q_{1}^{2} & =-Q_{1}^{2}, \quad q_{2}^{2}=-Q_{2}^{2}, \\
q_{3}^{2} & =-Q_{3}^{2}=-Q_{1}^{2}-2 Q_{1} Q_{2} \tau-Q_{2}^{2}, \quad q_{4}^{2}=0 .
\end{aligned}
$$

\section{Mandelstam representation}

Although the scalar functions in the master formula (6) are needed only for the reduced kinematics (7), where the limit $q_{4} \rightarrow 0$ is taken, we define the dispersion relation in the Mandelstam variables of the four-point function with general kinematics and evaluate it only afterwards for the special case $q_{4} \rightarrow 0$. This procedure has the following advantage: the HLbL contribution to $(g-2)_{\mu}$ splits into contributions from different topologies, each of them linked to a specific sub-process, which is either data input or again a dispersively reconstructed quantity. These different contributions are discussed in the following.

Gauge invariance, encoded in the decomposition (3), leads to Lorentz structures $T_{i}^{\mu \nu \lambda \sigma}$ of mass dimension 4 , 6 , and 8 . Hence, we expect the scalar functions $\Pi_{i}$ to be rather strongly suppressed at high energies. Thus we write down unsubtracted double-spectral (Mandelstam) representations for the $\Pi_{i}$ [26], i.e. parameter-free dispersion relations. The input to the dispersion relation are the residues at poles (due to single-particle intermediate states) and the discontinuities along branch cuts (due to two-particle intermediate states). Both are defined by the unitarity relation, in which the intermediate states are always on-shell. We neglect contributions from intermediate states consisting of more than two particles in the primary cut. Heavier intermediate states are expected to be suppressed by higher thresholds and smaller phase space, in agreement with the outcome of model calculations.
In the Mandelstam representation, the sum over intermediate states in the unitarity relations (for the primary and secondary cuts) translates into a splitting of the HLbL tensor into several topologies, shown in fig. 1. The first topology consists of the pion pole, i.e. the terms arising from a single pion intermediate state. This contribution is well-known [25] and given by

$$
\begin{aligned}
& \bar{\Pi}_{1}^{\pi^{0} \text {-pole }}=-\frac{\mathcal{F}_{\pi^{0} \gamma^{*} \gamma^{*}}\left(-Q_{1}^{2},-Q_{2}^{2}\right) \mathcal{F}_{\pi^{0} \gamma^{*} \gamma^{*}}\left(-Q_{3}^{2}, 0\right)}{Q_{3}^{2}+M_{\pi}^{2}}, \\
& \bar{\Pi}_{2}^{\pi^{0} \text {-pole }}=-\frac{\mathcal{F}_{\pi^{0} \gamma^{*} \gamma^{*}}\left(-Q_{1}^{2},-Q_{3}^{2}\right) \mathcal{F}_{\pi^{0} \gamma^{*} \gamma^{*}}\left(-Q_{2}^{2}, 0\right)}{Q_{2}^{2}+M_{\pi}^{2}},
\end{aligned}
$$

where $\mathcal{F}_{\pi^{0} \gamma^{*} \gamma^{*}}$ denotes the pion transition form factor (for off-shell photons but an on-shell pion).

The other topologies are obtained by selecting twopion intermediate states in the primary cut. The subprocess $\gamma^{*} \gamma^{*} \rightarrow \pi \pi$ is again cut in the crossed channel. If we single out the pion-pole contribution in both of the sub-processes, we obtain the box topologies for HLbL. For higher intermediate states in the crossed channel of $\gamma^{*} \gamma^{*} \rightarrow \pi \pi$, we obtain boxes with multi-particle cuts instead of poles in the sub-processes.

By explicitly constructing the Mandelstam representation, we have shown that the box topologies in the sense of unitarity have the same analytic structure as the oneloop sQED contribution, multiplied with pion electromagnetic form factors $F_{\pi}^{V}\left(q_{i}^{2}\right)$ for each of the off-shell photons (FsQED). The dispersion relation defines unambiguously this particular $q_{i}^{2}$ dependence. With the construction of the Mandelstam representation, we prove that FsQED and box topologies are the same. Note that the SQED loop contribution in terms of Feynman diagrams consists of boxes, triangles, and bulbs, but that the corresponding unitarity diagrams are just box topologies. This can be understood as follows: in SQED, the appearance of triangle and bulb diagrams is due to the seagull vertex, needed to ensure gauge invariance. In our formalism, gauge invariance is already encoded in the tensor decomposition (3). If the sQED contribution is projected on this tensor decomposition, which separates kinematics from dynamics, one can check that the dynamical singularities of the scalar functions $\Pi_{i}$ in SQED are the ones of a box topology.

The equivalence of the pion-box topologies with FsQED allows us to derive compact expressions for the contribution to the scalar functions $\Pi_{i}$ in terms of twodimensional Feynman parameter integrals. In the limit 
$q_{4} \rightarrow 0$, they are given by

$$
\begin{aligned}
\Pi_{i}^{\pi \text {-box }}\left(q_{1}^{2}, q_{2}^{2}, q_{3}^{2}\right)= & F_{\pi}^{V}\left(q_{1}^{2}\right) F_{\pi}^{V}\left(q_{2}^{2}\right) F_{\pi}^{V}\left(q_{3}^{2}\right) \\
& \times \frac{1}{16 \pi^{2}} \int_{0}^{1} d x \int_{0}^{1-x} d y I_{i}(x, y),
\end{aligned}
$$

where

$$
\begin{aligned}
I_{1}(x, y)= & -\frac{2}{3} \frac{(1-2 y)(1-2 x-2 y)(1-6 x(1-x))}{\Delta_{123}^{2}} \\
I_{4}(x, y)= & -\frac{2}{3} \frac{(1-2 x)(1+2 x(1-3 x(1-2 y)-6 y(1-y)))}{\Delta_{123}^{2}} \\
I_{7}(x, y)= & -\frac{4}{3} \frac{(1-2 x)^{2}(1-2 y)^{2} y(1-y)}{\Delta_{123}^{3}} \\
I_{16}(x, y)= & \frac{4}{3} \frac{x(1-2 x) y(1-2 y)}{\Delta_{213} \Delta_{13}}\left(\frac{1}{\Delta_{213}}+\frac{1}{\Delta_{13}}\right) \\
I_{19}(x, y)= & -\frac{4}{3} \frac{(1-2 x)^{2}(1-2 y) y(1-y)(1-2 x-2 y)}{\Delta_{213}^{3}} \\
I_{31}(x, y)= & -\frac{8}{3} \frac{x^{2}(1-x)(1-2 x)^{3} y(1-2 y)}{\Delta_{213} \Delta_{13}} \\
& \times\left(\frac{1}{\Delta_{213}^{2}}+\frac{1}{\Delta_{213} \Delta_{13}}+\frac{1}{\Delta_{13}^{2}}\right) \\
I_{39}(x, y)= & \frac{4}{3} \frac{(1-2 x)(1-2 y)^{2} y(1-y)(1-2 x-2 y)}{\Delta_{123}^{3}} \\
I_{42}(x, y)= & -\frac{4}{3} \frac{x(1-2 x) y(1-2 y)(1-6 y(1-y))}{\Delta_{321} \Delta_{21}} \\
I_{50}(x, y)= & 0, \\
& \left(\frac{1}{\Delta_{321}}+\frac{1}{\Delta_{21}}\right),
\end{aligned}
$$

and

$$
\begin{aligned}
\Delta_{i j k} & =M_{\pi}^{2}-x y q_{i}^{2}-x(1-x-y) q_{j}^{2}-y(1-x-y) q_{k}^{2} \\
\Delta_{i j} & =M_{\pi}^{2}-x(1-x) q_{i}^{2}-y(1-y) q_{j}^{2} .
\end{aligned}
$$

The remaining functions $\Pi_{i}$ that enter the master formula can be obtained with crossing relations permuting only $q_{1}$, $q_{2}$, and $q_{3}$, which are still valid in the limit $q_{4} \rightarrow 0$.

For a numerical analysis of the pion box contribution, the only input needed is the pion vector form factor in the space-like region. In fig. 2 we show two fits: one is based on a modified Omnès representation which accounts for the prominent singularities in the low-energy region $[27,28]$, the other on vector meson (here $\rho$-) dominance. Correspondently we obtain

$$
\begin{aligned}
a_{\mu}^{\pi \text { box }} & \simeq-15.9 \cdot 10^{-11} \\
a_{\mu}^{\pi \text { box,VMD }} & \simeq-16.4 \cdot 10^{-11}
\end{aligned}
$$

Error estimate is in progress but associated uncertainties are expected to be very small.

We treat the contribution from topologies with higher intermediate states in a partial-wave picture. This means that the a multi-particle cut is approximated by a polynomial, as illustrated in fig. 3. The dispersive formulation

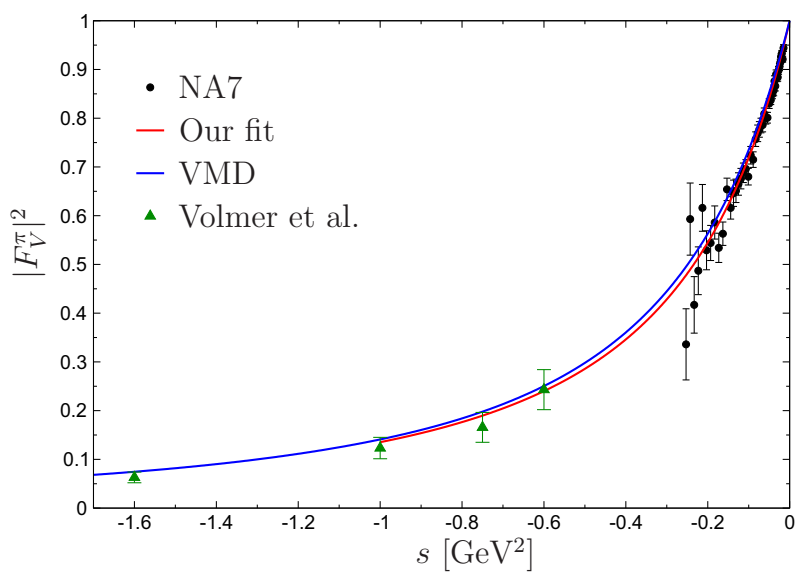

Figure 2. Different parameterization of the pion vector form factor for space-like kinematics used in our numerical analysis of the pion box contribution to $a_{\mu}$.

allows us to describe here the effect of two-pion rescattering in the primary channel. In [13], we already discussed the $S$-wave contribution. The new Lorentz decomposition allows us to include also higher partial waves. The contribution of these topologies is given by dispersion integrals over products of $\gamma^{*} \gamma^{(*)} \rightarrow \pi \pi$ helicity partial waves. The Born terms of the sub-process have to be properly subtracted to avoid double-counting with the box topologies.

\section{Conclusion and outlook}

Using the Mandelstam representation for the hadronic scalar functions $\Pi_{i}$, we have split $a_{\mu}^{\mathrm{HLbL}}$ into three contributions: pion-pole contributions, box topologies, and $\pi \pi$ rescattering contributions:

$$
a_{\mu}^{\mathrm{HLbL}}=a_{\mu}^{\pi^{0}-\text { pole }}+a_{\mu}^{\mathrm{box}}+a_{\mu}^{\pi \pi}+\ldots
$$

where the dots denote neglected higher intermediate states in the primary cut. We stress that in our approach we need to solve the dispersion relation for the HLbL tensor at fixed photon virtualities. The input quantities in our dispersive description are the pion transition form factor $\mathcal{F}_{\pi^{0} \gamma^{*} \gamma^{*}}\left(q_{i}^{2}, q_{j}^{2}\right)$, the pion electromagnetic form factor $F_{\pi}^{V}\left(q_{i}^{2}\right)$, and the $\gamma^{*} \gamma^{*} \rightarrow \pi \pi$ helicity partial waves. In the absence of experimental data on the doubly-virtual processes, these quantities will be reconstructed again dispersively [13, 29-36].

Our dispersive formalism defines unambiguously both the pion-pole and pion-box contribution. They are treated without any approximation. For the two-pion rescattering contribution a partial-wave expansion is employed.

We have limited the discussion to pions although the formalism can be extended to higher pseudoscalar poles $\left(\eta, \eta^{\prime}\right)$ or $K K$ intermediate states [37-40]. Future work will include model estimates of the contribution from intermediate states with more than two pseudoscalar particles, and the incorporation of high-energy constraints from perturbative QCD. 

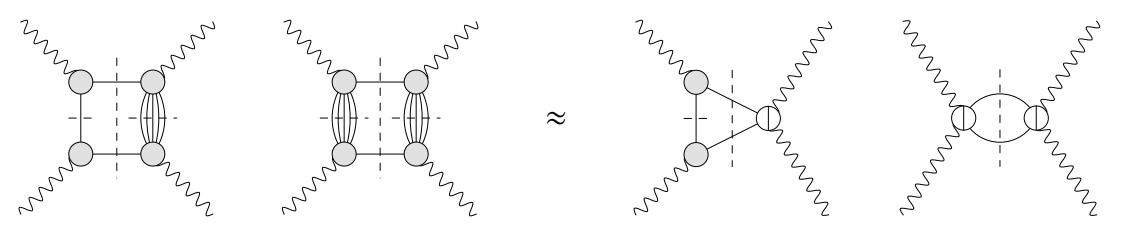

Figure 3. Partial-wave approximation of multi-particle intermediate states in the secondary cut.

Our dispersive approach is expected to help substantially reduce model dependence in HLbL scattering through a more data-driven evaluation of the HLbL contribution to $(g-2)_{\mu}$. A careful numerical study is currently under way, with the final goal to identify the experimental input with the largest impact on the theory uncertainty.

The speaker (MP) thanks the organizing committee of the Workshop FCCP2015 for the kind invitation. We thank J. Bijnens, J. Gasser, M. Knecht, B. Kubis, H. Leutwyler, A. Nyffeler, and S. Scherer for useful discussions. We gratefully acknowledge financial support by the Swiss National Science Foundation, the DFG (CRC 16, "Subnuclear Structure of Matter"), the European Community's 7th Framework Programme (Marie Curie IntraEuropean Fellowship) and the DOE (Grant No. DE-FG0200ER41132).

\section{References}

[1] G. W. Bennett et al. [Muon g-2 Collaboration], Phys. Rev. D 73 (2006) 072003 [hep-ex/0602035].

[2] T. Blum, A. Denig, I. Logashenko, E. de Rafael, B. Lee Roberts, T. Teubner and G. Venanzoni, arXiv:1311.2198 [hep-ph].

[3] J. Grange et al. [Muon g-2 Collaboration], arXiv:1501.06858 [physics.ins-det].

[4] N. Saito [J-PARC g-'2/EDM Collaboration], AIP Conf. Proc. 1467 (2012) 45.

[5] A. Kurz, T. Liu, P. Marquard and M. Steinhauser, Phys. Lett. B 734 (2014) 144 [arXiv:1403.6400 [hep$\mathrm{ph}]$.

[6] G. Colangelo, M. Hoferichter, A. Nyffeler, M. Passera and P. Stoffer, Phys. Lett. B 735 (2014) 90 [arXiv:1403.7512 [hep-ph]].

[7] C. Lehner, these proceedings.

[8] M. Hayakawa, T. Blum, T. Izubuchi and N. Yamada, PoS LAT 2005 (2006) 353 [hep-lat/0509016].

[9] T. Blum, M. Hayakawa and T. Izubuchi, PoS LATTICE 2012 (2012) 022 [arXiv:1301.2607 [hep-lat]].

[10] T. Blum, S. Chowdhury, M. Hayakawa and T. Izubuchi, Phys. Rev. Lett. 114 (2015) 1, 012001 [arXiv:1407.2923 [hep-lat]].

[11] J. Green, O. Gryniuk, G. von Hippel, H. B. Meyer and V. Pascalutsa, arXiv:1507.01577 [hep-lat].

[12] T. Blum, N. Christ, M. Hayakawa, T. Izubuchi, L. Jin and C. Lehner, arXiv:1510.07100 [hep-lat].
[13] G. Colangelo, M. Hoferichter, M. Procura and P. Stoffer, JHEP 1409 (2014) 091 [arXiv:1402.7081 [hep-ph]].

[14] G. Colangelo, M. Hoferichter, B. Kubis, M. Procura and P. Stoffer, Phys. Lett. B 738 (2014) 6 [arXiv:1408.2517 [hep-ph]].

[15] V. Pauk and M. Vanderhaeghen, Phys. Rev. D 90 (2014) 11, 113012 [arXiv:1409.0819 [hep-ph]].

[16] P. Stoffer, arXiv:1412.5171 [hep-ph].

[17] G. Colangelo, M. Hoferichter, M. Procura and P. Stoffer, JHEP 1509 (2015) 074 [arXiv:1506.01386 [hep-ph]].

[18] R. A. Leo, A. Minguzzi and G. Soliani, Nuovo Cim. A 30 (1975) 270.

[19] W. A. Bardeen and W. K. Tung, Phys. Rev. 173 (1968) 1423 [Phys. Rev. D 4 (1971) 3229].

[20] R. Tarrach, Nuovo Cim. A 28 (1975) 409.

[21] G. Eichmann, C. S. Fischer, W. Heupel and R. Williams, arXiv:1411.7876 [hep-ph].

[22] G. Eichmann, C. S. Fischer and W. Heupel, Phys. Rev. D 92 (2015) 5, 056006 [arXiv:1505.06336 [hep$\mathrm{ph}]]$.

[23] J. Aldins, T. Kinoshita, S. J. Brodsky and A. J. Dufner, Phys. Rev. D 1 (1970) 2378.

[24] J. L. Rosner, Annals Phys. 44 (1967) 11.

[25] M. Knecht and A. Nyffeler, Phys. Rev. D 65 (2002) 073034 [hep-ph/0111058].

[26] S. Mandelstam, Phys. Rev. 112 (1958) 1344.

[27] H. Leutwyler, hep-ph/0212324.

[28] G. Colangelo, Nucl. Phys. Proc. Suppl. 131 (2004) 185 [hep-ph/0312017].

[29] F. Niecknig, B. Kubis and S. P. Schneider, Eur. Phys. J. C 72 (2012) 2014 [arXiv:1203.2501 [hep-ph]].

[30] S. P. Schneider, B. Kubis and F. Niecknig, Phys. Rev. D 86 (2012) 054013 [arXiv:1206.3098 [hep-ph]].

[31] M. Hoferichter, G. Colangelo, M. Procura and P. Stoffer, Int. J. Mod. Phys. Conf. Ser. 35 (2014) 1460400 [arXiv:1309.6877 [hep-ph]].

[32] M. Hoferichter, B. Kubis, S. Leupold, F. Niecknig and S. P. Schneider, Eur. Phys. J. C 74 (2014) 3180 [arXiv:1410.4691 [hep-ph]].

[33] R. Garcia-Martin and B. Moussallam, Eur. Phys. J. C 70 (2010) 155 [arXiv:1006.5373 [hep-ph]].

[34] M. Hoferichter, D. R. Phillips and C. Schat, Eur. Phys. J. C 71 (2011) 1743 [arXiv:1106.4147 [hep-ph]].

[35] B. Moussallam, Eur. Phys. J. C 73 (2013) 2539 [arXiv:1305.3143 [hep-ph]]. 
[36] M. Hoferichter, B. Kubis and D. Sakkas, Phys. Rev. D 86 (2012) 116009 [arXiv:1210.6793 [hep-ph]].

[37] F. Stollenwerk, C. Hanhart, A. Kupsc, U. G. Meissner and A. Wirzba, Phys. Lett. B 707 (2012) 184 [arXiv:1108.2419 [nucl-th]].

[38] C. Hanhart, A. Kup?c, U.-G. Meißner, F. Stollenwerk and A. Wirzba, Eur. Phys. J. C 73 (2013) 12, 2668 [Eur.
Phys. J. C 75 (2015) 6, 242] [arXiv:1307.5654 [hep$\mathrm{ph}]$.

[39] B. Kubis and J. Plenter, Eur. Phys. J. C 75 (2015) 6, 283 [arXiv:1504.02588 [hep-ph]].

[40] C. W. Xiao, T. Dato, C. Hanhart, B. Kubis, U.G. Meißner and A. Wirzba, arXiv:1509.02194 [hep$\mathrm{ph}$. 